\title{
Suppression of Surface-Related Loss in a Gated Semiconductor Microcavity
}

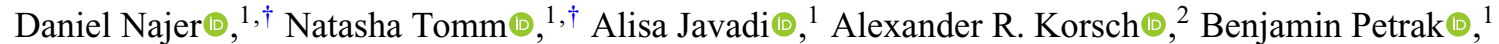 \\ Daniel Riedelø, ${ }^{1}$ Vincent Dolique $\odot,{ }^{3}$ Sascha R. Valentin $\odot,{ }^{2}$ Rüdiger Schott $\odot,{ }^{2}$ Andreas D. Wieck $\odot,{ }^{2}$ \\ Arne Ludwig $\odot,{ }^{2}$ and Richard J. Warburton $\odot^{1, *}$ \\ ${ }^{1}$ Department of Physics, University of Basel, Klingelbergstrasse 82, 4056 Basel, Switzerland \\ ${ }^{2}$ Lehrstuhl für Angewandte Festkörperphysik, Ruhr-Universität Bochum, 44780 Bochum, Germany \\ ${ }^{3}$ Laboratoire des Matériaux Avancés (LMA), IN2P3/CNRS, Université de Lyon, Villeurbanne 69622, Lyon, France
}

(Received 9 December 2020; revised 17 February 2021; accepted 25 February 2021; published 1 April 2021)

\begin{abstract}
We present a surface-passivation method that reduces surface-related losses by almost 2 orders of magnitude in a highly miniaturized GaAs open microcavity. The microcavity consists of a curved dielectric distributed Bragg reflector with radius of approximately $10 \mu \mathrm{m}$ paired with a GaAs-based heterostructure. The heterostructure consists of a semiconductor distributed Bragg reflector followed by an $n-i-p$ diode with a layer of quantum dots in the intrinsic region. Free-carrier absorption in the highly- $n$-doped and highly- $p$-doped layers is minimized by our positioning them close to a node of the vacuum electromagnetic field. The surface, however, resides at an antinode of the vacuum field and results in significant loss. These losses are much reduced by surface passivation. The strong dependence on wavelength implies that the main effect of the surface passivation is to eliminate the surface electric field, thereby quenching below-band-gap absorption via a Franz-Keldysh-like effect. An additional benefit is that the surface passivation reduces scattering at the GaAs surface. These results are important in other nanophotonic devices that rely on a GaAs-vacuum interface to confine the electromagnetic field.
\end{abstract}

DOI: 10.1103/PhysRevApplied.15.044004

\section{INTRODUCTION}

Concepts in cavity quantum electrodynamics (QED) can be implemented with use of semiconductors. A semiconductor-based microcavity can be created with a micropillar [1-3], a photonic crystal cavity [4,5], a whispering-gallery resonator [6], and an open microcavity [7-9]. Quantum dots (QDs) within these structures mimic atoms. In the limit of a single QD, a single-photon source can be realized by exploiting the weak-coupling regime of cavity QED [10]. The strong-coupling regime of cavity QED has been accessed with three different microcavity platforms $[1,4,9]$.

In all these semiconductor-based applications of cavity QED, minimizing the absorption and scattering losses in the microcavity is very important. For single-photon sources operating in the weak-coupling regime, efficient

*richard.warburton@unibas.ch

†These authors contributed equally to this work.

Published by the American Physical Society under the terms of the Creative Commons Attribution 4.0 International license. Further distribution of this work must maintain attribution to the author(s) and the published article's title, journal citation, and DOI. photon extraction from the microcavity is essential $[2,3]$. In the strong-coupling regime, a coherent exchange between an exciton in the QD and a photon in the microcavity is possible only if the exciton-photon coupling exceeds the rate of photon loss. Typically, this requires the development of low-mode-volume, high- $Q$-factor microcavities. A recurring theme in the development of such microcavities is the role of the GaAs surface. At the semiconductor surface, the symmetry of the lattice is broken. The GaAs surface is quite complex - there are a number of possible surface reconstructions - and a thin oxide layer typically forms on exposure to air [11]. Reducing surface-related absorption is crucial in the development of GaAs-based cavity QED.

Recently, considerable success in implementing cavity QED was reported with a QD in an open microcavity $[7-9,12]$. The "bottom" mirror is a semiconductor distributed Bragg reflector (DBR) and the "top" mirror is a curved dielectric DBR. The position of the bottom mirror can be tuned in situ with respect to the position of the top mirror, allowing a single QD to be brought into resonance with the microcavity mode. In the latest developments, the QDs are embedded in an $n-i-p$ diode $[9,12]$. Both $n$-doped GaAs and $p$-doped GaAs result in freecarrier absorption [13]. To minimize the absorption within the microcavity, the $n$-doped and $p$-doped layers are made 
as thin as possible and are positioned close to the node of the vacuum electric field. This technique, positioning an absorbing layer at a node of the vacuum electric field, can also be used to reduce losses at the GaAs surface. But in this case it involves a serious compromise. If there is a node at the surface, the largest vacuum electric field lies in the vacuum gap and not in the GaAs material on account of interferences in the device. This reduces considerably the coupling of a QD to the vacuum electric field. An acceptably large coupling is possible only if there is a vacuum field antinode at the surface. Success with $n-i-p$ devices in an open microcavity was possible only after passivation of the surface $[9,12]$. The role played by the passivation is elucidated here.

We probe the surface-related absorption in an open microcavity. The main diagnostic tool is a measurement of the wavelength dependence of the $Q$ factor. With an untreated surface, we find that the $Q$ factor is modest, approximately $10^{4}$ at the stopband (SB) center, much lower than the value expected from the mirror designs. Following surface passivation, we find that the $Q$ factor increases to approximately $10^{6}$ at the stopband center, close to the value expected from the mirror designs. This shows, first, that the dominant loss mechanism in the untreated case is related to the GaAs surface and, second, that surface passivation remedies this loss. For the untreated surface, the $Q$ factor has a very strong dependence on wavelength, increasing rapidly on tuning to lower wavelengths. By comparing the $Q$ factor with the result of model calculations, we find that we can account quantitatively for the $Q$ factor by ascribing the loss to absorption in the capping layer, the final 55-nmthick GaAs layer of the heterostructure. The absorption in the capping layer is an exponential function of the photon energy, pointing to Franz-Keldysh-like absorption induced by a strong electric field at the surface [14-18]. In turn, this demonstrates the main role of the surface-passivation layer in this device: it reduces the surface electric field, thereby much reducing the Franz-Keldysh absorption. The standard analytic result for the Franz-Keldysh effect describes the absorption at the unpassivated surface but with an electric field much higher than in the standard picture (midgap pinning).

\section{THE OPEN, TUNABLE MICROCAVITY}

The microcavity $[7,19]$ consists of a curved dielectric DBR - the template is produced by $\mathrm{CO}_{2}$-laser ablation [20] - paired with a "nip-DBR" semiconductor heterostructure. InAs QDs are embedded in the intrinsic part of the $n-i-p$ diode; the diode resides on top of a semiconductor DBR [Fig. 1(a)]. We use two top dielectric DBRs. The first (DBR I) is composed of 22 pairs of $\mathrm{SiO}_{2}(\lambda / 4)$ and $\mathrm{Ta}_{2} \mathrm{O}_{5}(\lambda / 4)$ (where $\lambda$ depicts the wavelength in each material) and is terminated with $\mathrm{SiO}_{2}$; the SB center [21] is at $973 \mathrm{~nm}$. The second (DBR II) is composed of 15 pairs of

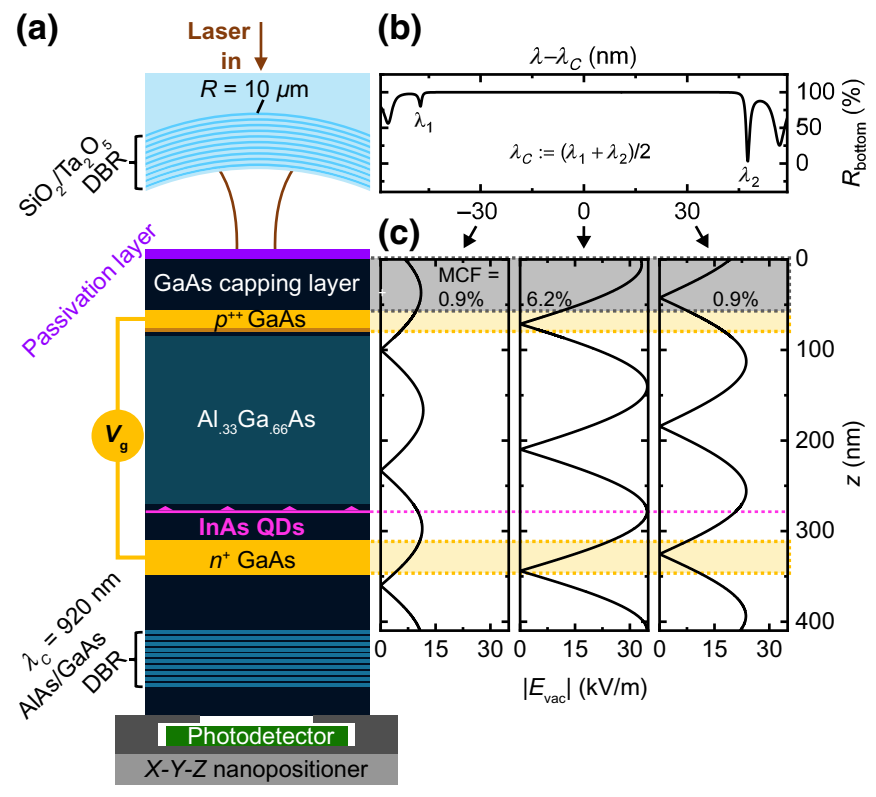

FIG. 1. Ultrahigh- $Q$-factor optical microcavity as a sensitive probe of surface-related losses. (a) Schematic of the microcavity involving a curved dielectric DBR and an $n-i-p$ heterostructure with self-assembled InAs QDs on top of a semiconductor DBR ("nip-DBR"). (b) Simulated reflectance of the nip-DBR with SB center $\lambda_{C}=920 \mathrm{~nm}$. (c) Calculated vacuum field amplitude across the heterostructure for three different wavelengths, -30 , 0 , and $+30 \mathrm{~nm}$ with respect to the SB center. As the antinodes of the vacuum field shift in position with wavelength, thereby changing the MCF in the GaAs capping layer, surface-related absorption in the capping layer $\left(10^{-10}-10^{-8} \mathrm{~cm}^{-1}\right)$ can be probed via the microcavity by our measuring its $Q$ factor across the SB. At $\lambda_{C}$, where the coupling to the QDs is maximized, free-carrier absorption in the highly- $p$-doped and highly- $n$-doped gates is minimized by our placing them close to a vacuum field node. Note also that the highly reduced vacuum field at $\lambda-\lambda_{C}=$ $-30 \mathrm{~nm}$ arises because at this wavelength the largest vacuum electric field is located in the vacuum gap.

$\mathrm{SiO}_{2}(\lambda / 4)$ and $\mathrm{Ta}_{2} \mathrm{O}_{5}(\lambda / 4)$ and is terminated with $\mathrm{Ta}_{2} \mathrm{O}_{5}$; the SB center is at $930 \mathrm{~nm}$. The semiconductor DBR consists of 46 pairs of $\operatorname{AlAs}(\lambda / 4)$ and $\operatorname{GaAs}(\lambda / 4)$. The heterostructure is a $1.5 \lambda$ layer of GaAs including doped layers acting as a top gate $\left(p^{++}, 10^{19} \mathrm{~cm}^{-3}\right)$ and a back gate $\left(n^{+}\right.$, $\left.2 \times 10^{18} \mathrm{~cm}^{-3}\right)$. The QD layer is placed at an antinode of the vacuum electric field (at a distance $\lambda$ below the surface). The intrinsic region between QDs and the back gate acts as tunnel barrier for electrons and ensures that the QDs operate under Coulomb blockade at low temperature [22]. Using a piezoelectric-based $X-Y-Z$ nanopositioner, the microcavity features full in situ tunability at cryogenic temperatures.

A measurement of the $Q$ factor across the SB of the nip-DBR [Fig. 1(b)] reveals possible sources of loss in the heterostructure because the standing wave inside the cavity shifts with wavelength [Fig. 1(c)]. For instance, losses 
in the capping layer depend on the exact wavelength: at a wavelength detuning of $-30,0$, and $+30 \mathrm{~nm}$ with respect to the nip-DBR's SB center, the calculated modal confinement factor (MCF; defined as the vacuum electromagnetic energy confined in the layer of interest divided by the zeropoint energy, $\hbar \omega / 2$, i.e., the total energy of the vacuum field mode) of the capping layer is $0.9 \%, 6.2 \%$, and $0.9 \%$, respectively. Therefore, if the dominant loss mechanism in the microcavity occurs within the capping layer, then the change in MCF will result in a strong dependence of the $Q$ factor across the stopband. Furthermore, by careful characterization of the mirrors and by simulation of the entire structure with transfer-matrix calculations, measurements of the $Q$ factor not only reveal the location of the dominant loss process but can also be used to determine the loss quantitatively.

\section{GaAs SURFACE PASSIVATION}

Surface passivation of GaAs replaces the native oxide with a thin $\mathrm{Al}_{2} \mathrm{O}_{3}$ layer $[6,23]$. The surface-passivation recipe follows in the first part of the procedures described in Ref. [24]. As a first cleaning step, the processed semiconductor sample (already containing Au contact pads) is successively immersed in acetone, 2-propanol, and ethanol inside an ultrasonic bath at $40^{\circ} \mathrm{C}$. To prevent surface treatment of the contact pads, they are covered with a manually applied drop of photoresist (AZ1512HS, Microchemicals $\mathrm{GmbH}$ ) and baked for $10 \mathrm{~min}$ at $100^{\circ} \mathrm{C}$. At room temperature, the sample is dipped into an $\mathrm{HCl}$ solution (25\%) for $1 \mathrm{~min}$ to remove the native oxide $[24,25]$. The sample is then rinsed with deionized water for approximately $1 \mathrm{~s}$ and immediately immersed in an $\left(\mathrm{NH}_{4}\right)_{2} \mathrm{~S}$ solution $(20 \%)$ for $10 \mathrm{~min}$. This procedure passivates the surface with sulfur [26,27], preventing the native oxide from reforming. The sulfur layer is, however, not robust. For this reason, it is removed and replaced with an $\mathrm{Al}_{2} \mathrm{O}_{3}$ layer. To achieve this, on its emerging from the $\left(\mathrm{NH}_{4}\right)_{2} \mathrm{~S}$ solution, the sample is blown dry with nitrogen and immediately transferred into an atomic-layer-deposition (ALD) chamber (Savannah 100, Cambridge NanoTech Inc.).

The following ALD recipe is chosen to deposit approximately $8 \mathrm{~nm}$ of $\mathrm{Al}_{2} \mathrm{O}_{3}$ onto the sample surface: $T=$ $150^{\circ} \mathrm{C}$, first pulse $50 \mathrm{~ms}$ (water), wait for $12 \mathrm{~s}$, second pulse $40 \mathrm{~ms}$ (trimethylaluminium), wait for $10 \mathrm{~s}$; the cycle is repeated 80 times. The $\mathrm{Al}_{2} \mathrm{O}_{3}$ layer acts as a diffusion barrier for oxygen [29], thus preventing reoxidation of the etched GaAs surface.

After surface passivation, the remaining challenge is to remove the photoresist that has been cross-linked due to the high temperature, $150{ }^{\circ} \mathrm{C}$, inside the ALD chamber. The use of $N$-methyl-2-pyrrolidone (NMP) at elevated temperatures is shown to remove successfully the crosslinked photoresist. The sample is immersed in NMP for
9-20 $\mathrm{h}\left(20 \mathrm{~h}\right.$ yields the best result) at $40{ }^{\circ} \mathrm{C}$ and then successively cleaned for $5 \mathrm{~min}$ in NMP, acetone, 2-propanol, and methanol in an ultrasonic bath at approximately $56^{\circ} \mathrm{C}$. As a final step, a polymeric strip coating (First Contact, Photonic Cleaning Technologies) is used to remove any final residues from the sample surface.

\section{INDIVIDUAL-MIRROR CHARACTERIZATION}

Each DBR has high reflectance for wavelengths within the stopband. Outside the stopband, there are oscillations in the reflectivity as a function of wavelength. These oscillations are sensitive to the exact layer thicknesses in the particular mirror - this dependence is exploited to characterize the layers in each DBR.

As depicted in Fig. 2, the cavity's top and bottom mirrors are characterized at $4.2 \mathrm{~K}$ by a broadband light source (white-light-emitting-diode or halogen lamp) and a darkfield confocal microscope [28]. The light from the source is coupled into a single-mode optical fiber, the output of which is collimated and focused onto the sample surface with an objective lens (NA 0.55). Cross-polarizing elements are used in the beam path to reject all but the light reflected from the sample surface. The detection fiber is connected to a spectrometer [28]. The reflected light from a metallic mirror, the Au contact pad in the case of the nip-DBR, is used to record a reference spectrum. The nipDBR's reflectance spectrum is obtained by dividing its reflected spectrum by the reference spectrum. Because of the absence of a metallic reference surface on top of dielectric DBR I in Fig. 2(a), an exponential fit of the reference spectrum from Fig. 2(b) is used instead, and the maximum reflectance is normalized to 1 .

Via one-dimensional (1D) transfer-matrix methods (ESSENTIAL MACLEOD, Thin Film Center Inc.) the design layer thicknesses can be refined to fit the reflectivity oscillations outside the stopband. The models obtained for each DBR (solid red lines in Fig. 2) provide a convincing description of these oscillations. These mirror descriptions are then used to simulate the cavity performance, in particular the $Q$ factors and transmittance values at resonance as a function of the vacuum gap between the mirrors (see Sec. IX). The slight discrepancy between experiment and the model arises from the difficulty of recording precisely a reference spectrum for the white-light source.

\section{MICROCAVITY CHARACTERIZATION: $Q$ FACTORS}

A microcavity is constructed with use of passivated and unpassivated semiconductor DBRs and a curved dielectric DBR (radius of curvature approximately 7-16 $\mu \mathrm{m}$ ) similar to the ones characterized in Fig. 2. Via narrowband-laser transmission measurements, each microcavity is characterized by our determining its $Q$ factor across the SB of the semiconductor DBR. The transmission signal is measured 
(a)

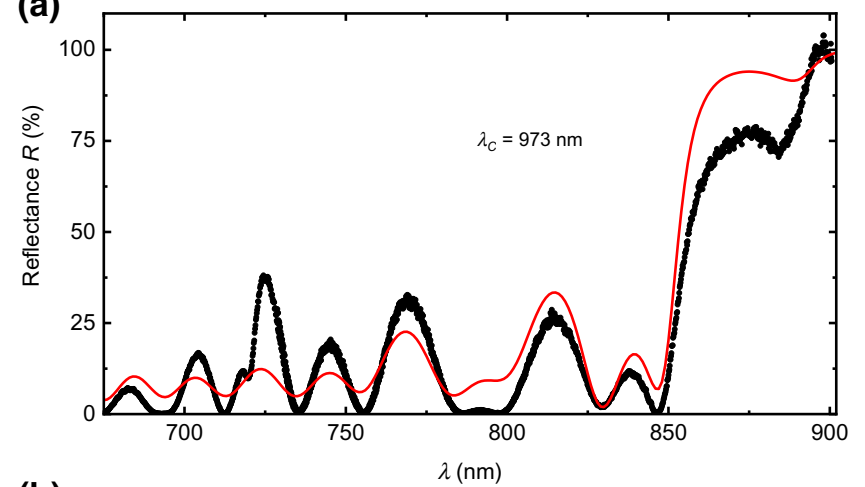

(b)

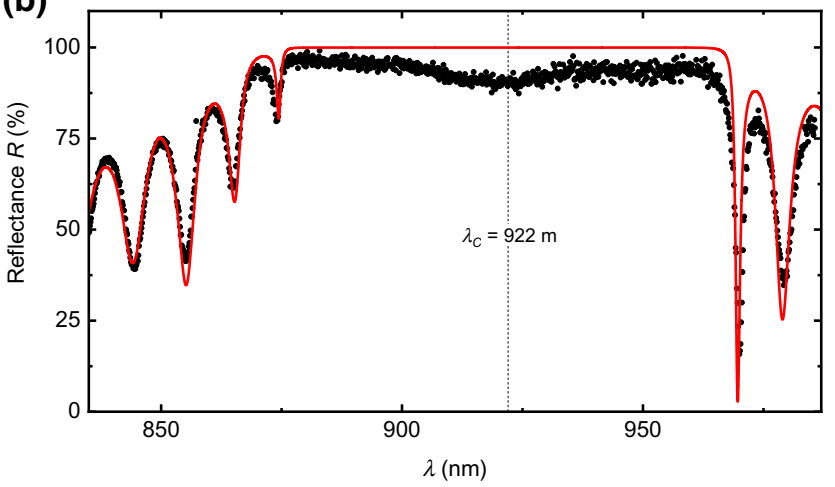

FIG. 2. Mirror characterization via reflection measurements. Each mirror is investigated by our recording the spectrum of white light reflected off the sample using a dark-field confocal microscope [28]. Via 1D transfer-matrix methods (ESSENTIAL MACLEOD), the designed layer thicknesses can be refined to fit the experimentally observed oscillations. (a) Dielectric DBR I. The reflected signal is recorded on a flat surface away from the curved part of the mirror and normalized by the white-light spectrum. (b) Unpassivated nip-DBR. Here the reflectance spectrum is obtained by our normalizing the signal reflected from the mirror by the signal reflected from a Au contact pad (by our moving the piezoelectric nanopositioner laterally by a few microns).

as a function of laser frequency with our keeping the cavity length fixed [Fig. 3(a)]. To change the cavity's resonance frequency, the mirror separation is changed by means of the $Z$ nanopositioner.

A $Q$ factor is obtained for every pair of longitudinal $\left(\mathrm{TEM}_{00}\right)$ modes at the minimum mirror separation (approximately $2-4 \mu \mathrm{m}$, depending on the wavelength and mirror-crater depth [19]) by our fitting a double Lorentzian. Figures 3(a) and 3(b) show the results for an electrically contacted passivated sample (black dots), an electrically uncontacted unpassivated sample (blue triangles), and an electrically contacted unpassivated sample (red squares), paired with DBR I as the top mirror [30]. Without passivation, the $Q$ factor is around $10^{5}$ for the electrically uncontacted unpassivated sample at the SB center, and is too low to measure precisely for the electrically contacted unpassivated sample. At a redshift of (a)
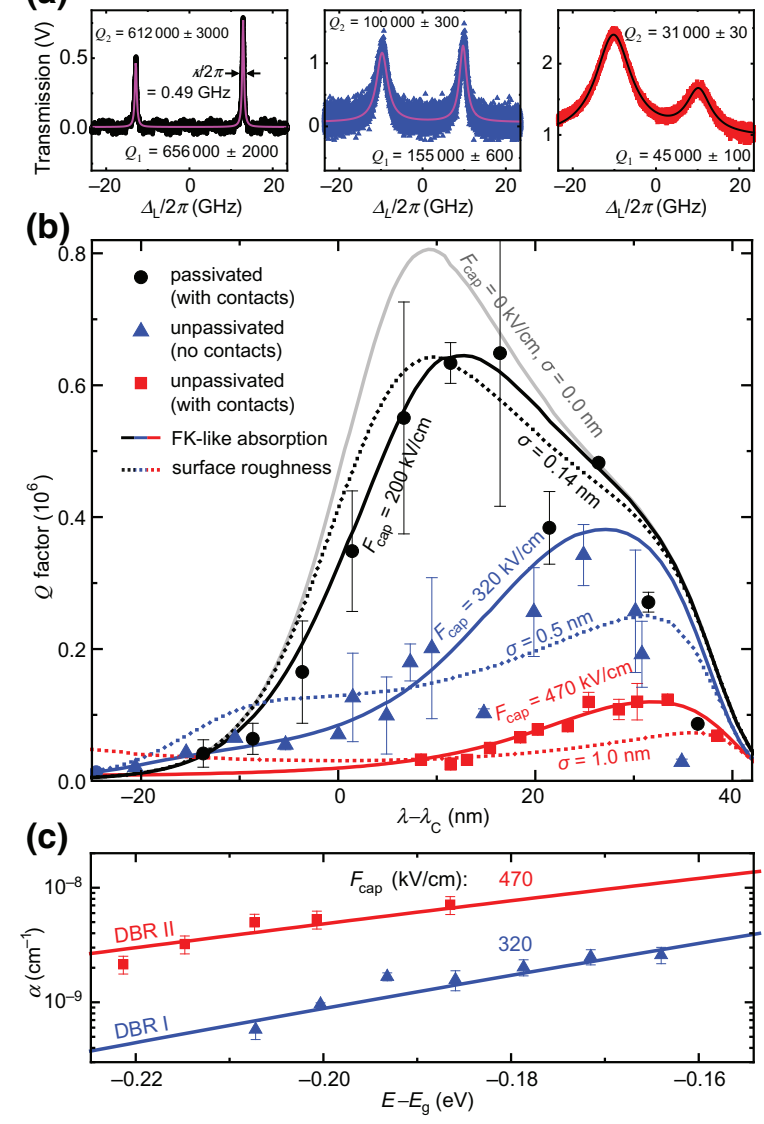

FIG. 3. Microcavity characterization via $Q$-factor measurements using DBR I paired with passivated nip-DBR with contacts (black dots), two unpassivated nip-DBRs without contacts (blue triangles), and DBR I (DBR II) paired with five (one) unpassivated DBRs with contacts (red squares). (a) Measured transmission signal as a function of laser detuning at $\lambda-\lambda_{C} \sim 10 \mathrm{~nm}$ at fixed mirror separation. The $Q$ factor is determined by a double-Lorentzian fit (solid lines). (b) Evaluated $Q$ factors for several wavelengths. Mean values and standard deviations originate from data from two cavity modes and up to six measurements. Two surface-loss mechanisms are modeled: absorption and scattering. The solid lines are calculated $Q$ factors with our taking into account free-carrier absorption (absorption coefficients from Ref. [13]) and Franz-Keldysh (FK) absorption $[17,18]$ for electric fields in the capping layer $F_{\text {cap }}=$ $(200,320,470) \mathrm{kV} / \mathrm{cm}$. The dotted lines are calculated by our taking into account surface scattering only [31]. For the passivated case, the dotted black line is modeled with roughness at both the GaAs-alumina interface and the alumina-vacuum interface $\sigma=\left(\sigma_{\mathrm{GaAs}-\mathrm{Al}_{2} \mathrm{O}_{3}}, \sigma_{\mathrm{Al}_{2} \mathrm{O}_{3} \text {-vac }}\right)$, and is found to be $\sigma=$ $(0.14,0.14) \mathrm{nm}$; alternatively $\sigma=(0.0,0.55) \mathrm{nm}$ yields similar results. In the unpassivated cases, the dotted blue and red lines are modeled with $\sigma_{\mathrm{GaAs} \text {-vac }}=0.5 \mathrm{~nm}$ and $\sigma_{\mathrm{GaAs} \text {-vac }}=1.0 \mathrm{~nm}$ at the GaAs-vacuum interface, respectively. The solid gray line is the model without any surface losses. (c) By comparing measured and simulated $Q$ factors, and assuming that the scattering losses are negligible, one can deduce the absorption coefficient $\alpha$ as a function of photoenergy. $\alpha$ is fitted to the Franz-Keldysh result with $F_{\text {cap }}$ as a fitting parameter. 
$10 \mathrm{~nm}$ from the SB center, the $Q$ factor is on average $3.8 \times 10^{4}$ for the electrically contacted unpassivated sample and $1.28 \times 10^{5}$ for the electrically uncontacted unpassivated sample [Fig. 3(a)]. These values are much smaller than the values expected from the DBRs - they signify that there is a significant loss mechanism. Around the SB center, where the coupling to the QD layer is maximized $\left(\lambda_{C}=915-925 \mathrm{~nm}\right)$, the $Q$ factors are strongly decreased by this loss mechanism - the loss impacts significantly the performance of a QD in the microcavity.

As the wavelength approaches the red end of the SB, the $Q$ factors for the unpassivated samples increase. There is a pronounced asymmetry with respect to the SB center: the $Q$ factors decrease monotonically as the wavelength shifts to the blue with respect to the SB center. These results imply that the loss mechanism is a strong function of wavelength.

After passivation, the $Q$ factor around the SB center increases. At a redshift of $10 \mathrm{~nm}$ from the SB center, the $Q$ factor increases to a very large value, $6.34 \times 10^{5}$ [Fig. 3(a)]. Furthermore, the dependence of the $Q$ factor on wavelength is much more symmetric with respect to the SB center. The decrease on the blue side reflects the decrease in reflectivity of the dielectric mirror, which, for fabrication reasons, has a maximum reflectivity at a wavelength of $973 \mathrm{~nm}$.

The passivation procedure changes the properties of the surface but leaves the rest of the microcavity unchanged. The drastic increase of the $Q$ factors after surface passivation leads therefore to the conclusion that the losses limiting the $Q$ factors of unpassivated microcavities are related to the semiconductor surface. Specifically, the loss, either an absorption or a scattering mechanism, originates at the GaAs surface itself or in the GaAs layer immediately below the surface.

These results are verified in a second experiment using a different piece of wafer material from the nip-DBR. The passivation is performed in a separate run; the cavity is constructed with DBR II as the top mirror. The results are shown in Fig. 4. The results follow closely those of the first experiment, with the advantage that the $Q$ factors can be determined also on the blue side of the SB center.

To quantify the loss, the entire microcavity is modeled (see Sec. IX) using accurate descriptions of the two DBRs, including the free-carrier absorption in the doped layers in the heterostructure. Absorption is added to the capping layer (GaAs between the $p$ doping and the surface) and adjusted to match the experimentally determined $Q$ factors at each wavelength. This is a robust procedure as the surface-related loss dominates other loss channels. The extracted absorption coefficients $\alpha$ from this procedure are plotted as a function of wavelength in Fig. 3(c).

In both cases shown in Fig. 3(c) the absorption coefficients $\alpha$ are extremely small. From a measurement point of view, the microcavity represents a very sensitive platform

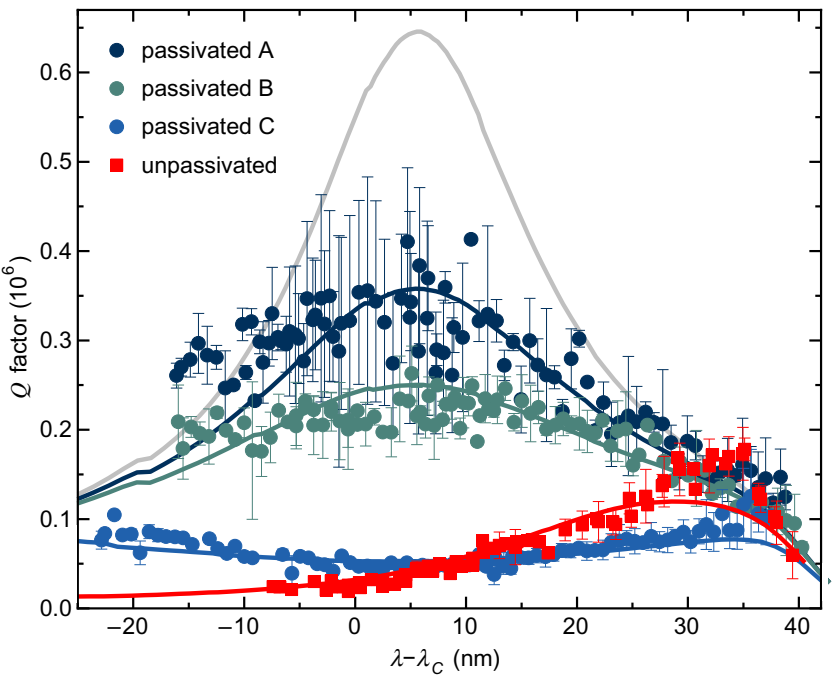

FIG. 4. Determination of surface-related losses via $Q$-factor measurements using DBR II with a nip-DBR. We probe the double role of surface passivation. Four positions in the contacted sample are probed: three passivated areas, A, B, and C (circles), and an unpassivated region (red squares). Mean values and standard deviation result from the measurements on the two cavity modes. We implement a mixed model to fit the data: Franz-Keldysh-like absorption and reasonable values of surface roughness are probed together. For the passivated regions, surface roughness alone explains the observed data. We estimate a roughness $\sigma=\left(\sigma_{\mathrm{GaAs}-\mathrm{Al}_{2} \mathrm{O}_{3}}, \sigma_{\mathrm{Al}_{2} \mathrm{O}_{3} \text {-vac }}\right)$ for each region: at region A $\sigma=(0.5,0.5) \mathrm{nm}$, alternatively $\sigma=(0.0,1.0) \mathrm{nm}$; at region B $\sigma=(0.25,0.8) \mathrm{nm}$, alternatively $\sigma=(0.0,1.4) \mathrm{nm}$; at region $\mathrm{C} \sigma=(0.5,3.0) \mathrm{nm}$, alternatively $\sigma=(0.0,3.75) \mathrm{nm}$. For the unpassivated region, surface roughness alone cannot account for the $Q$-factor dependence on wavelength alone: with a surface roughness of $\sigma_{\mathrm{GaAs} \text {-vac }}=0.3 \mathrm{~nm}$, an electric field $F_{\text {cap }}=400$ $\mathrm{kV} / \mathrm{cm}$ is still needed to fit the data.

for detecting very weak absorption or scattering events. In a single-pass experiment, these losses would be very difficult to detect. Significantly, we find that $\alpha$ depends exponentially on photon energy in the unpassivated case, a dependence that rules out scattering or broadband absorption as the main loss mechanism at the surface, as these processes would have a much weaker dependence on wavelength. Instead, the exponential dependence points to below-gap absorption in an electric field.

\section{INVESTIGATION OF LOSS VIA SURFACE ROUGHNESS}

To confirm that the measured losses on the heterostructure with doping are indeed a consequence of the doped layers and to quantify losses via surface scattering, we compare our results with those for a microcavity consisting of a semiconductor heterostructure without doping. The heterostructure in this case is a $\lambda$ layer of GaAs 

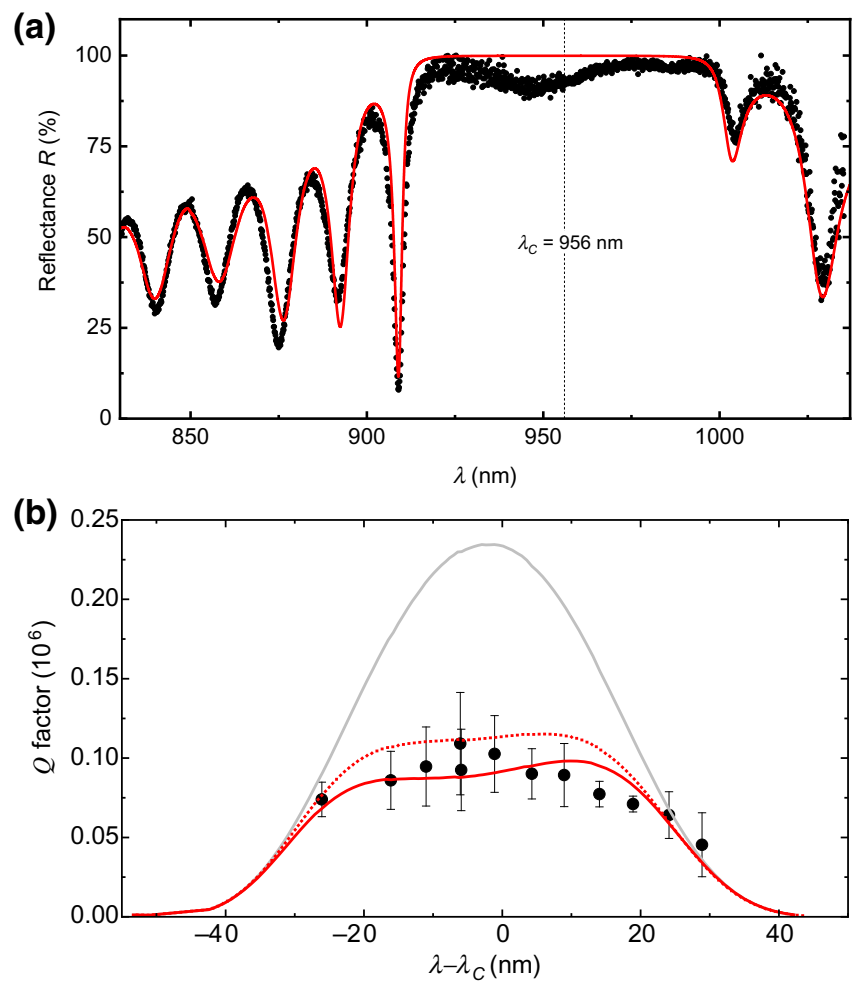

FIG. 5. Undoped semiconductor heterostructure: reflectance and $Q$-factor measurements. (a) Reflectance measurement of a semiconductor heterostructure without doping $\left(\lambda_{C}=956 \mathrm{~nm}\right)$ at $4.2 \mathrm{~K}$. The heterostructure contains a $\lambda$ layer of GaAs on top of 33 pairs of $\operatorname{AlAs}(\lambda / 4) / \operatorname{GaAs}(\lambda / 4)$. A layer of QDs is included at the center of the GaAs layer. To record a reference spectrum, parts of the sample are covered by a Au film by means of electron-beam evaporation. (b) $Q$ factor of a microcavity at $4.2 \mathrm{~K}$ consisting of this heterostructure without doping paired with dielectric top mirror DBR I: experimental results (circles) and simulation with surface roughness $\sigma_{\mathrm{GaAs} \text {-vac }}=0.5 \mathrm{~nm}$ (solid red line). The dotted red line is the expected $Q$ factor upon passivation of the surface with a 8.0 -nm-thick $\mathrm{Al}_{2} \mathrm{O}_{3}$ layer. The solid gray line corresponds to expected $Q$ factor without any surface losses.

(with embedded InAs QDs in the center) on top of a 33pair AlAs/GaAs DBR [7,8,32]. It is a high-quality sample but may not match the ultrahigh quality of the semiconductor heterostructure with doping. Initially, we repeat the mirror-characterization procedure described above and find a suitable model for the semiconductor layer thicknesses [Fig. 5(a)]. Subsequently, we pair this mirror with dielectric top mirror DBR I.

Figure 5(b) depicts the measured $Q$ factors as a function of wavelength for the semiconductor heterostructure without doping. The $Q$ factor reaches approximately $10^{5}$ at the stopband center, and remains constant within measurement error over a wavelength range of approximately $20 \mathrm{~nm}$. This behavior is quite different from that of the unpassivated semiconductor heterostructure with doping [Figs. 3(b) and 3(c) and 4]. The conclusion is that the strongly-wavelength-dependent loss process is related to the doping.

In the absence of losses, the heterostructure-withoutdoping-top-mirror combination should yield a $\mathrm{Q}$ factor of $2 \times 10^{5}$ in the stopband center [Fig. 5(b)], solid gray line], about a factor of 2 higher than that determined experimentally [Fig. 5(b), black circles]. The wavelength dependence of the $Q$ factor [Fig. 5(b)] is, as before, a useful diagnostic of the scattering process. We find that in this case scattering alone at the GaAs-vacuum interface can account for the measured $Q$ factors, with surface roughness $\sigma_{\mathrm{GaAs} \text {-vac }}=$ $0.5 \mathrm{~nm}$ [Fig. 5(b), solid red line]. We simulate as well the possible outcome of passivating the undoped sample, with $\sigma=\left(\sigma_{\mathrm{GaAs}-\mathrm{Al}_{2} \mathrm{O}_{3}}, \sigma_{\mathrm{Al}_{2} \mathrm{O}_{3} \text {-vac }}\right)=(0.5,0.5) \mathrm{nm}$, shown in Fig. 5(b) as the dotted red line: the addition of a thin alumina layer would diminish the effect of surface scattering due to surface roughness, increasing slightly the expected $Q$ factor.

A surface roughness $\sigma$ translates into a total integrated scatter of approximately $(4 \pi \sigma / \lambda)^{2}$ [33], and can be modeled by an extinction coefficient $k$ (for the 1D transfer-matrix methods) according to Ref. [31],

$$
k=\frac{\pi\left(n_{1}-n_{2}\right)^{2}\left(n_{1}+n_{2}\right) d}{\lambda \sqrt{8\left(n_{1}^{2}+n_{2}^{2}\right)}},
$$

where $n_{1}$ and $n_{2}$ are the refractive indices of the two layers surrounding the scattering layer of thickness $d=$ $2 \sigma$, and $\lambda$ is the free-space wavelength. Including this loss in the simulations reproduces the measured $Q$ factors convincingly. Furthermore, atomic-force-microscopy (AFM) measurements (tapping mode, Bruker Dimension 3100 ) indicate that surface roughness is present in different amounts across the samples, as shown in Fig. 6. The undoped wafer has root-mean-square surface roughness $0.3 \mathrm{~nm} \leq \sigma \leq 0.7 \mathrm{~nm}$, the unpassivated bare sample (without contacts) has $0.2 \mathrm{~nm} \leq \sigma \leq 0.9 \mathrm{~nm}$, the unpassivated contacted sample has $0.4 \mathrm{~nm} \leq \sigma \leq 7.3 \mathrm{~nm}$, and the passivated sample has $0.3 \mathrm{~nm} \leq \sigma \leq 1.9 \mathrm{~nm}$. The native surface roughness at the GaAs-vacuum interface, immediately after growth of the samples, is on the order of $0.15-0.30 \mathrm{~nm}$. However, increased roughness is caused by processing of the samples (e.g., via passivation, cleaning and gluing, and bonding). We speculate that in the unpassivated contacted samples, the high values of surface roughness might be induced by remnant traces of photoresist from the processing procedure (which in turn have a lower scattering power than pure GaAs, due to the reduced refractive index).

The passivated doped sample also provides an opportunity to test the applicability of the total integrated scatter result for loss at a rough interface. The $Q$ factors measured with DBR II at three different passivated regions of the sample exhibit a weak dependence on wavelength, 
(a)

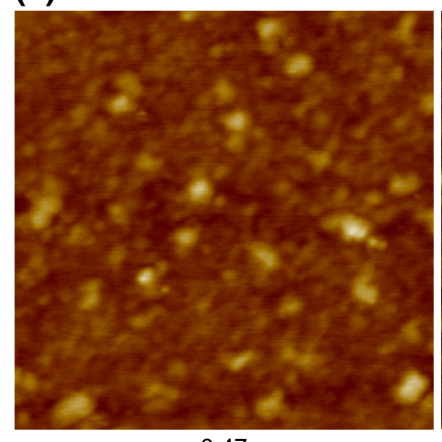

$\sigma=0.47 \mathrm{~nm}$ (b)

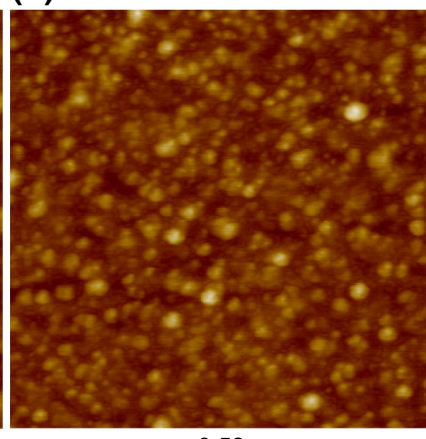

(c)

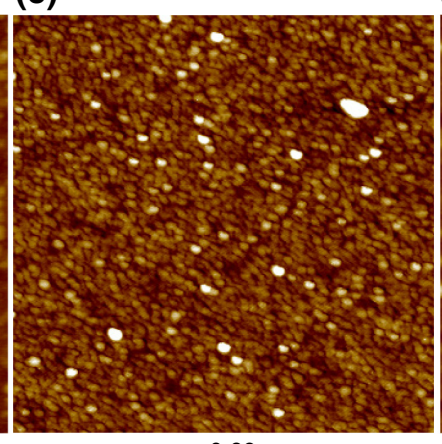

(d)

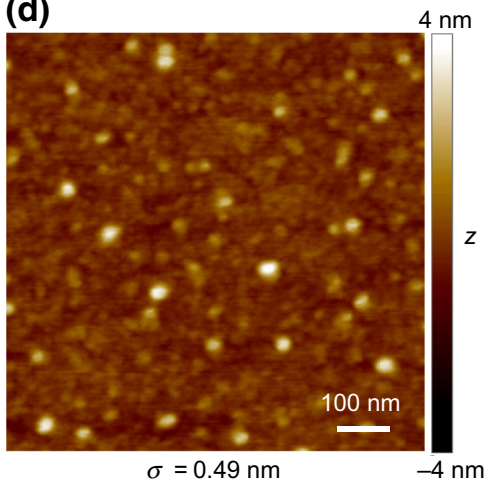

FIG. 6. AFM images of different semiconductor samples. Each image depicts the same scan area of $0.8 \times 0.8 \mu \mathrm{m}^{2}(\mathrm{obtained} \mathrm{in}$ tapping mode) and from a height of -4 to $+4 \mathrm{~nm}$. (a) Piece of passivated wafer. The root-mean-square surface roughness measured at several similar sample locations lies in the range $0.3 \mathrm{~nm} \leq \sigma \leq 1.9 \mathrm{~nm}$. (b) Piece of unpassivated bare wafer. The range of surface roughness for similar sample locations is $0.3 \mathrm{~nm} \leq \sigma \leq 0.7 \mathrm{~nm}$. (c) Unpassivated sample with contacts. Similar samples exhibit $0.4 \mathrm{~nm} \leq \sigma \leq 7.3 \mathrm{~nm}$. (d) Piece of unpassivated bare wafer from a semiconductor DBR without $n$-type and $p$-type layers. Different sample locations exhibited surface roughness in the range $0.2 \mathrm{~nm} \leq \sigma \leq 0.9 \mathrm{~nm}$.

in contrast to the results in the unpassivated region. The model with $\sigma=(0.5,0.5) \mathrm{nm}, \sigma=(0.25,0.8) \mathrm{nm}$, and $\sigma=(0.5,3.0) \mathrm{nm}$ for regions $\mathrm{A}, \mathrm{B}$, and $\mathrm{C}$, respectively, reproduces these $Q$ factors precisely, with reasonable values of roughness at each interface (Fig. 4). Furthermore, the model captures the symmetric influence of roughness on the $Q$ factor with respect to the SB center. This results from the reduction in the MCF at the sample's surface, as presented in Fig. 1(c). Agreement between the $Q$ factors and the simulation gives us confidence that the description of loss via surface scattering is quantitatively correct.

The conclusion from this measurement and analysis is that passivation of the surface not only quenches surfacerelated absorption but also has the beneficial effect of reducing loss via surface scattering. The original GaAs surface and the passivated surface have a similar range of values of surface roughness. In the passivated case, the surface loss via scattering is about $60 \%$ with respect to that of the unpassivated sample. The passivation procedure creates a layer with an intermediate refractive index: it avoids the large jump in refractive index at a GaAs-vacuum interface. This reduces the total scattering loss.

In the case of the doped unpassivated sample, the wavelength dependence of the $Q$ factors is too strong to be accounted for by scattering loss [Figs. 3(b) and 4]. A different mechanism is clearly at play.

\section{MICROSCOPIC EXPLANATION FOR THE NIP-DBR LOSSES}

We give a possible microscopic explanation for the losses in the investigated nip-DBR structure and why surface passivation significantly reduces them. In Fig. 7(a), the calculated valence-band and conduction-band edges in the heterostructure are shown, a solution to the 1D Poisson equation (obtained via nextnano software, nextnanomat, nextnano $\mathrm{GmbH}$ ). In the unpassivated case, we simulate the midgap Fermi-level pinning at the surface via a Schottky barrier of $0.76 \mathrm{eV}$. This yields an electric field in the capping layer ("capping field") $F_{\text {cap }}$ of $140 \mathrm{kV} / \mathrm{cm}$.

An electric field in a semiconductor leads to FranzKeldysh absorption below the band gap of the material $[14,15]$ : owing to the position dependence of the band edges, the electron and hole wave functions can be described by Airy functions (similar to a particle in a triangular well [34]) and acquire an exponential tail at energies within the band gap. The electric field therefore creates an absorption processes at photon energies $E_{\text {photon }}<E_{g}$. The situation is schematically depicted in Fig. 7(b).

According to the standard model [16], Franz-Keldysh absorption at photon energy $E$ due to the presence of an electric field $F$ can be described via the absorption coefficient:

$$
\begin{aligned}
\alpha(E, F)= & \beta \frac{F^{1 / 3}}{E} \sum_{i=\mathrm{lh}, \mathrm{hh}}\left(\frac{\mu_{i}}{m_{0}}\right)^{4 / 3}\left|M_{i}\right|^{2} \\
& \times\left[\left|\mathrm{Ai}^{\prime}\left(x_{i}\right)\right|^{2}-x_{i}\left|\operatorname{Ai}\left(x_{i}\right)\right|^{2}\right],
\end{aligned}
$$

where

$$
x_{i}=\frac{e\left(E_{g}-E\right)}{\hbar \theta_{i}}
$$

and

$$
\hbar \theta_{i}=\left[\frac{(e F \hbar)^{2}}{2 \mu_{i}}\right]^{1 / 3} .
$$

In these equations, $\beta$ is a constant (arbitrary units), $e$ is the elementary charge (in SI units), $\hbar$ is the 

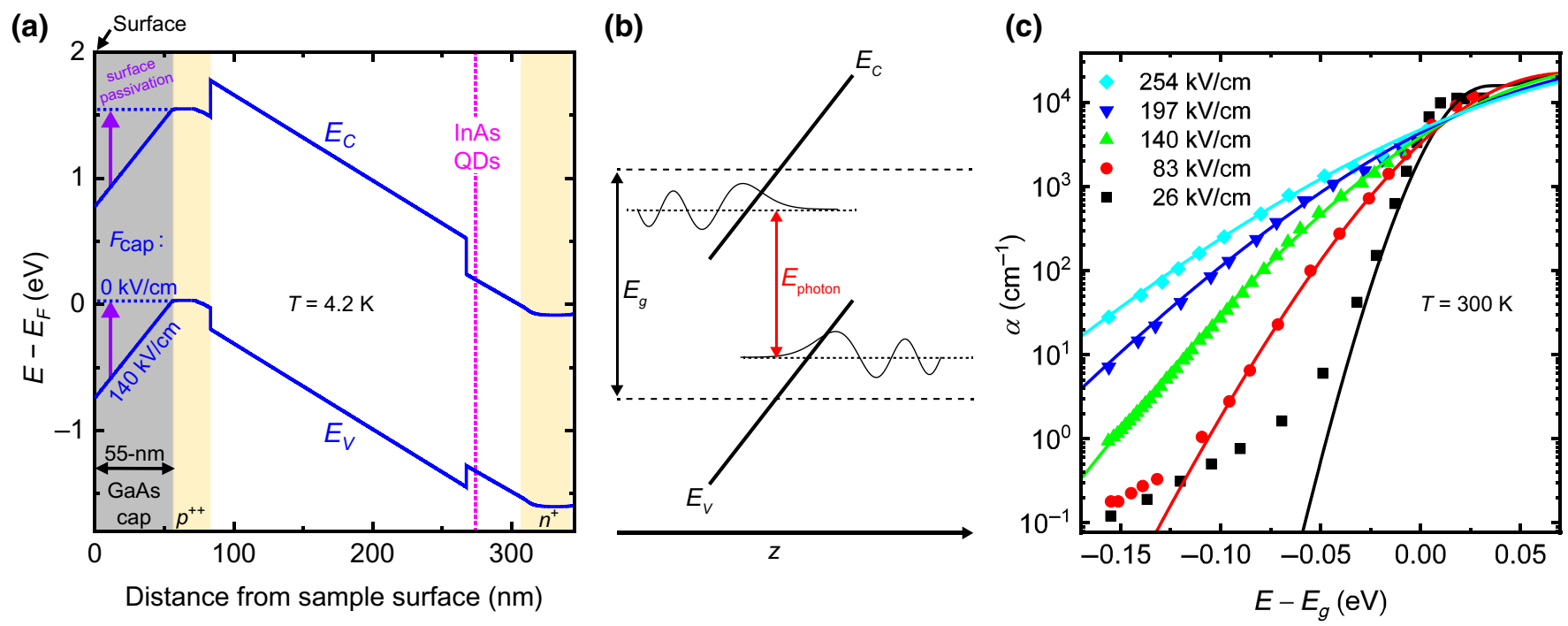

FIG. 7. Band structure and Franz-Keldysh effect. (a) Simulation of the conduction and valence bands in the $n-i-p$ diode (nextnanomat) at $4.2 \mathrm{~K}$. The surface is modeled via a Schottky barrier of $E_{g} / 2=0.76 \mathrm{eV}$ reflecting the midgap Fermi-level pinning at the GaAs surface [24]. The effect of surface passivation is to eliminate the electric field at the capping layer. (b) Schematic of the Franz-Keldysh effect [14,15]. An electric field applied to a semiconductor allows both electrons and holes to tunnel into the forbidden energy, leading to below-gap absorption processes. (c) Room-temperature Franz-Keldysh absorption coefficients for different electric fields within a $p-i-n$ double heterostructure (IMG 2020 IEEE. Reprinted, with permission, from Ref. [18]). The solid lines correspond to the calculated absorption coefficients according to Refs. [16,17] [Eqs. (2)-(6)].

reduced Planck constant (in SI units), $m_{0}$ is the free electron rest mass (in kilograms), $\mu_{\mathrm{lh}}=0.037 m_{0}\left(\mu_{\mathrm{hh}}=\right.$ $\left.0.058 m_{0}\right)$ is the reduced mass of an electron-light-hole pair (electron-heavy-hole pair), $F$ is given in kilovolts per centimeter, the energies $E_{g}$ and $E$ are given in electronvolts, and $\left|M_{\mathrm{lh}}\right|^{2}\left(\left|M_{\mathrm{hh}}\right|^{2}\right)$ the momentum matrix elements for the light-hole (heavy-hole). Ai(xi) is an Airy function [35].

We use of the momentum matrix elements derived in Ref. [17] for different polarizations of the radiation field. For light polarized in the $x-y$ plane, the momentum matrix elements for the light and heavy holes read

$$
\begin{gathered}
\left|M_{\mathrm{lh}}\right|^{2}=P^{2} / 3, \\
\left|M_{\mathrm{hh}}\right|^{2}=P^{2},
\end{gathered}
$$

where $P=0.692$ (arbitrary units) is a typical value for GaAs [17].

We use this model for Franz-Keldysh absorption to describe previously reported room-temperature experiments on a $p-i-n$ double heterostructure [18] [Fig. 7(c)], and extract the value of $\beta$ in Eq. (2), which is found to be $2.5 \times 10^{4}$ (arbitrary units). These experiments extend to photon energies far below the band gap, the case of interest here. There is a compelling overlap between theory and experiment.

To estimate Franz-Keldysh absorption coefficients in our nip-DBR at $4.2 \mathrm{~K}$ [Figs. 3(b) and 3(c) and 4], we use Eqs. (2)-(6), taking the low-temperature GaAs band gap of
$1.519 \mathrm{eV}$ and $\beta$ extracted from fitting the data in Fig. 7(c). We compare the results of the model for low-temperature Franz-Keldysh absorption with the experimental data presented in Figs. 3(b) and 3(c), taking the electric field $F$ as a fitting parameter. The exponential dependence of the absorption on the photon energy is well described by Franz-Keldysh absorption. However, in the unpassivated case, the capping field $F_{\text {cap }}=470 \mathrm{kV} / \mathrm{cm}$ when we do not account for any surface roughness (Fig. 3) and $F_{\text {cap }}=400$ $\mathrm{kV} / \mathrm{cm}$ when we account for a realistic value of surface roughness (Fig. 4). These values are, respectively 3.4 and 2.8 times greater than the values expected from the $1 \mathrm{D}$ Poisson equation [Fig. 7(a)]. The origin of this discrepancy is not understood at this time, but we note several points.

First, there are no Franz-Keldysh-absorption experiments reported in the literature at low temperature $(4.2 \mathrm{~K})$ and at photon energies far below the band gap $E_{g}$ of GaAs (at $E-E_{g} \sim-0.17 \mathrm{eV}$, corresponding to $\lambda \sim 920 \mathrm{~nm}$ ). Our approach here is to fit the theory presented in Refs. $[16,17]$ to the room-temperature experimental results reported in Ref. [18] [Fig. 7(c)] and then to extrapolate the absorption coefficients to photon energies approximately $0.17 \mathrm{eV}$ below the band gap. The change in temperature, from room temperature to low temperature, is accommodated by a rigid shift in the absorption spectrum to account for the increase in the band gap. It is conceivable that the standard Franz-Keldysh theory is inadequate at photon energies far below the band gap - this point has not been tested experimentally. 
Secondly, there are room-temperature experiments on Franz-Keldysh oscillations in doped GaAs heterostructures (a 25-80-nm-thick, undoped GaAs capping layer on top of an $n^{+}$-doped $\mathrm{Al}_{0.32} \mathrm{Ga}_{0.68}$ As layer [36]) that also report surface electric field values a factor 1.8-3.8 greater than the expected ones [37].

Thirdly, the Franz-Keldysh model describes a bulk semiconductor in a uniform electric field. Obviously, it does not take into account the microscopic details of the surface; for instance, surface reconstructions and oxidation. It is possible that the details of the surface layer are important here.

In the light of this analysis, our proposal is that surface passivation quenches surface-related absorption primarily by reducing the electric field in the capping layer, thereby eliminating the Franz-Keldysh absorption (within the sensitivity of the experiment).

A remaining question is why the bare-wafer sample without passivation shows higher $Q$ factors than the electrically contacted sample also without passivation [Figs. $3(\mathrm{~b})$ and 3(c)]. The contacting process may change the surface roughness, as revealed by the AFM measurements (Fig. 6). The surface roughness per se does not, however, account for the $Q$ factors of the various samples. The main point is that surface scattering does not account for the exponential dependence of the loss process on photon energy [Figs. 3(b) and 3(c)]. Instead, we speculate that the change in GaAs surface on forming the contacts results in a change of surface pinning, thereby increasing the capping field. One possibility is that the degradation of the surface on contacting spreads the available surface states to lower energies.

\section{MODEL FOR THE CURVED DIELECTRIC MIRRORS}

An interpretation of the microcavity $Q$ factors in terms of losses in the semiconductor heterostructure rests on an understanding of the top mirror. The top mirrors, dielectric DBRs, are of very high quality with very low loss. To prove this point, we investigate a microcavity formed from DBR I and a planar version of DBR I. The coatings for the plane mirror and concave mirrors are applied to the substrates in the same run and are nominally identical. Figure 8 shows the measured $Q$ factors. At the stopband center of the top mirror $\left(\lambda_{C}=976 \mathrm{~nm}\right)$, the $Q$ factor is extremely high, $1.5 \times 10^{6}$. To describe the dielectric mirror accurately at the stopband center of the semiconductor DBR, we analyze the dependence of the dielectric-dielectric $Q$ factor and transmission as a function of wavelength. To describe the high transmission at short wavelengths, we are forced to shift the stopband center of the bottom mirror by $3 \mathrm{~nm}$ to the red [38]. A rough interface at the "lower" surface of the five-"lowest" $\mathrm{Ta}_{2} \mathrm{O}_{5}$ layers shown

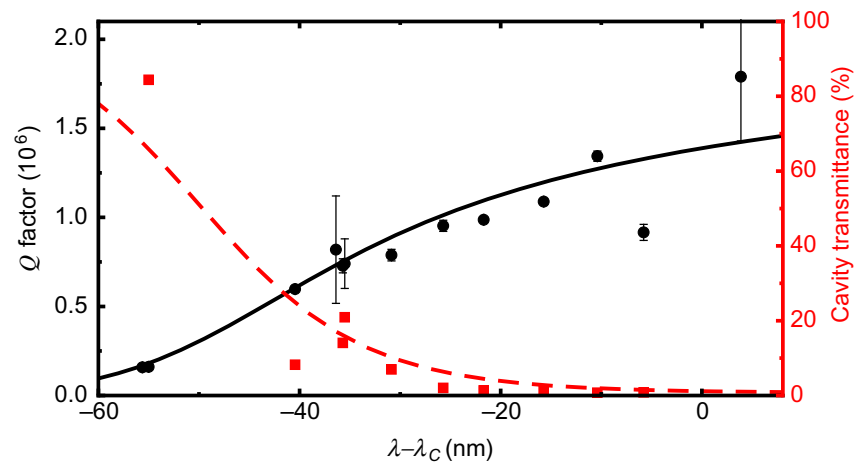

FIG. 8. Measured $Q$ factors and cavity transmittance of a purely dielectric microcavity. A dielectric top mirror $\left(\lambda_{C}=\right.$ $973 \mathrm{~nm}$ ) paired with a dielectric bottom mirror (shifted to $\lambda_{C}=$ $976 \mathrm{~nm}$, nominally the same coating) at $300 \mathrm{~K}$. The cavity transmittance is measured by our relating the transmitted power at the cavity resonance to the laser power before the objective lens multiplied by a fitted in-coupling efficiency of 59\%. The solid black line (dashed red line) corresponds to the calculated $Q$ factor (cavity transmittance) with our taking into account material extinction coefficients $k_{\mathrm{SiO}_{2}}=4 \times 10^{-7}$ and $k_{\mathrm{Ta}_{2} \mathrm{O}_{5}}=4.5 \times$ $10^{-7}$ [39]. Additionally, an interface roughness of $\sigma=0.25 \mathrm{~nm}$ above each of the five-last-grown $\mathrm{Ta}_{2} \mathrm{O}_{5}$ layers and $k=4 k_{\mathrm{Ta}_{2} \mathrm{O}_{5}}$ in the last-grown $\mathrm{Ta}_{2} \mathrm{O}_{5}$ layer are introduced heuristically to fit the experimental data.

in Fig. 1 (extinction coefficients corresponding to an interface roughness of $0.25 \mathrm{~nm}$ [31]) and increased absorption within the "lowest" $\mathrm{Ta}_{2} \mathrm{O}_{5}$ layer (extinction coefficient $k=$ $4 k_{\mathrm{Ta}_{2} \mathrm{O}_{5}}$, where $k_{\mathrm{Ta}_{2} \mathrm{O}_{5}}$ is defined in Sec. IX) are heuristically introduced in the model so as to describe the measured $Q$ factors. This fit is very convincing (Fig. 8). This description of dielectric top mirror DBR I is used to interpret the measurements on microcavities formed with the semiconductor DBRs as the bottom mirror [Figs. 3(b) and 3(c) and 5(b)].

\section{1D TRANSFER-MATRIX CALCULATION OF THE MICROCAVITY $Q$ FACTORS}

We list the relevant parameters used for the $Q$-factor calculations via a one-dimensional transfer-matrix method.

The extinction coefficients in the different materials are as follows. For DBR I, $k_{\mathrm{SiO}_{2}}=4 \times 10^{-7}$ [39], $k_{\mathrm{Ta}_{2} \mathrm{O}_{5}}=$ $4.5 \times 10^{-7}$ [39], and $k=4 k_{\mathrm{Ta}_{2} \mathrm{O}_{5}}$ is used for the "lowest" (i.e., the last-grown) $\mathrm{Ta}_{2} \mathrm{O}_{5}$ layer (the layer closest to the vacuum gap). For DBR II, $k_{\mathrm{SiO}_{2}}=4 \times 10^{-6}$ and $k_{\mathrm{Ta}_{2} \mathrm{O}_{5}}=4.5 \times 10^{-6}$ (from fitting data taken with curved DBR II and planar DBR I microcavity). For the semiconductor nip-DBR, $k_{p++}=5.2 \times 10^{-4}$ for $p^{++}$-doped GaAs, $k_{p+}=1.9 \times 10^{-4}$ for $p^{+}$-doped GaAs, and $k_{n+}=$ $0.7 \times 10^{-4}$ for $n^{+}$-doped GaAs [13]. An extinction coefficient of $k=\alpha_{\mathrm{FK}} \lambda /(4 \pi)$, where $\alpha_{\mathrm{FK}}$ is a Franz-Keldysh absorption coefficient, is introduced in the capping layer; $\alpha_{\mathrm{FK}}$ depends on the electric field. Surface roughness is 
described by our introducing an additional layer of thickness $d=2 \sigma$, where $\sigma$ is the root-mean-square surface or interface roughness [31].

In analogy to the experiment, a $Q$ factor is determined for a fixed "vacuum-gap" layer thickness by our calculating a cavity transmittance spectrum. A Lorentzian fit to the calculated spectrum is used to determine the resonance frequency as well as the $Q$ factor. This procedure is repeated for different vacuum gaps, yielding a plot of $Q$ factor versus wavelength. The resulting $Q$ factors are presented in Figs. $3-5$ and 8.

\section{CONCLUSION}

Significant surface-related losses in an open microcavity consisting of a nip-DBR and a dielectric DBR are much reduced by passivation of the GaAs surface. The passivation works primarily by eliminating the FranzKeldysh-like absorption in the capping layer. A secondary benefit of the passivation is to reduce loss by surface scattering. With passivation, $Q$ factors close to $10^{6}$ can be achieved.

\section{ACKNOWLEDGMENTS}

We thank Ivan Favero for inspiration and we thank Leonardo Midolo and Peter Lodahl for fruitful discussions. We thank Monica Schönenberger and the Nano Imaging Lab (University of Basel) for support acquiring AFM images. This work was funded by Swiss National Science Foundation project 200020_175748, NCCR QSIT and the project QLUSTER. A.J. acknowledges support from the European Union Horizon 2020 Research and Innovation Programme under Marie Skłodowska-Curie Grant Agreement No. 840453 (HiFig). S.R.V., R.S., A.L., and A.D.W. gratefully acknowledge support from DFH/UFA CDFA0506, DFG TRR160, DFG project 383065199, and BMBF Q.Link.X.

[1] J. Reithmaier, G. Sek, A. Löffler, C. Hofmann, S. Kuhn, S. Reitzenstein, L. Keldysh, V. Kulakovskii, T. Reinecke, and A. Forchel, Strong coupling in a single quantum dot-semiconductor microcavity system, Nature 432, 197 (2004).

[2] N. Somaschi, V. Giesz, L. De Santis, J. C. Loredo, M. P. Almeida, G. Hornecker, S. L. Portalupi, T. Grange, C. Anton, J. Demory, C. Gomez, I. Sagnes, N. D. LanzillottiKimura, A. Lemaitre, A. Auffeves, A. G. White, L. Lanco, and P. Senellart, Near-optimal single-photon sources in the solid state, Nat. Photonics 10, 340 (2016).

[3] X. Ding, Y. He, Z.-C. Duan, N. Gregersen, M.-C. Chen, S. Unsleber, S. Maier, C. Schneider, M. Kamp, S. Höfling, C.-Y. Lu, and J.-W. Pan, On-Demand Single Photons with High Extraction Efficiency and Near-Unity
Indistinguishability from a Resonantly Driven Quantum dot in a Micropillar, Phys. Rev. Lett. 116, 020401 (2016).

[4] T. Yoshie, A. Scherer, J. Hendrickson, G. Khitrova, H. Gibbs, G. Rupper, C. Ell, O. Shchekin, and D. Deppe, Vacuum Rabi splitting with a single quantum dot in a photonic crystal nanocavity, Nature 432, 200 (2004).

[5] K. Kuruma, Y. Ota, M. Kakuda, S. Iwamoto, and Y. Arakawa, Surface-passivated high- $q$ GaAs photonic crystal nanocavity with quantum dots, APL Photonics 5, 046106 (2020).

[6] B. Guha, F. Marsault, F. Cadiz, L. Morgenroth, V. Ulin, V. Berkovitz, A. Lemaitre, C. Gomez, A. Amo, S. Combrie, B. Gerard, G. Leo, and I. Favero, Surface-enhanced gallium arsenide photonic resonator with quality factor of $6 \times 10^{6}$, Optica 4, 218 (2017).

[7] R. J. Barbour, P. A. Dalgarno, A. Curran, K. M. Nowak, H. J. Baker, D. R. Hall, N. G. Stoltz, P. M. Petroff, and R. J. Warburton, A tunable microcavity, J. Appl. Phys. 110, 053107 (2011).

[8] L. Greuter, S. Starosielec, A. V. Kuhlmann, and R. J. Warburton, Towards high-cooperativity strong coupling of a quantum dot in a tunable microcavity, Phys. Rev. B 92, 045302 (2015).

[9] D. Najer, I. Söllner, P. Sekatski, V. Dolique, M. C. Löbl, D. Riedel, R. Schott, S. Starosielec, S. R. Valentin, A. D. Wieck, N. Sangouard, A. Ludwig, and R. J. Warburton, A gated quantum dot strongly coupled to an optical microcavity, Nature 575, 622 (2019).

[10] P. Senellart, G. Solomon, and A. White, High-performance semiconductor quantum-dot single-photon sources, Nat. Nanotechnol. 12, 1026 (2017).

[11] C. M. Demanet and M. A. Marais, A multilayer model for GaAs oxides formed at room temperature in air as deduced from an XPS analysis, Surf. Interface Anal. 7, 13 (1985).

[12] N. Tomm, A. Javadi, N. O. Antoniadis, D. Najer, M. C. Löbl, A. R. Korsch, R. Schott, S. R. Valentin, A. D. Wieck, A. Ludwig, and R. J. Warburton, A bright and fast source of coherent single photons, Nat. Nanotechnol. 1 (2021).

[13] H. C. Casey, D. D. Sell, and K. W. Wecht, Concentration dependence of the absorption coefficient for $n$ - and $p$-type GaAs between 1.3 and $1.6 \mathrm{eV}$, J. Appl. Phys. 46, 250 (1975).

[14] W. Franz, Einfluß eines elektrischen Feldes auf eine optische Absorptionskante, Z. Naturforsch., A 13, 484 (1958).

[15] V. L. Keldysh, Behaviour of non-metallic crystals in strong electric fields, J. Exp. Theor. Phys. 33, 994 (1957).

[16] D. E. Aspnes, Electric-field effects on optical absorption near thresholds in solids, Phys. Rev. 147, 554 (1966).

[17] J. Hader, N. Linder, and G. H. Döhler, $k \cdot p$ theory of the Franz-Keldysh effect, Phys. Rev. B 55, 6960 (1997).

[18] B. Knupfer, P. Kiesel, M. Kneissl, S. Dankowski, N. Linder, G. Weimann, and G. H. Dohler, Polarization-insensitive high-contrast GaAs/AlGaAs waveguide modulator based on the Franz-Keldysh effect, IEEE Photonics Technol. Lett. 5, 1386 (1993).

[19] L. Greuter, S. Starosielec, D. Najer, A. Ludwig, L. Duempelmann, D. Rohner, and R. J. Warburton, A small mode volume tunable microcavity: Development and characterization, Appl. Phys. Lett. 105, 121105 (2014).

[20] D. Hunger, C. Deutsch, R. J. Barbour, R. J. Warburton, and J. Reichel, Laser micro-fabrication of concave, 
low-roughness features in silica, AIP Adv. 2, 012119 (2012).

[21] In this work, we define the SB center as the mean value of the two wavelengths at the local minima (with $R<90 \%$ ) of the calculated reflectance spectrum that are closest to the maximum mirror reflectance [Fig. 1(b)].

[22] R. J. Warburton, C. Schaflein, D. Haft, F. Bickel, A. Lorke, K. Karrai, J. M. Garcia, W. Schoenfeld, and P. M. Petroff, Optical emission from a charge-tunable quantum ring, Nature 405, 926 (2000).

[23] J. Liu, K. Konthasinghe, M. Davanço, J. Lawall, V. Anant, V. Verma, R. Mirin, S. W. Nam, J. D. Song, B. Ma, Z. S. Chen, H. Q. Ni, Z. C. Niu, and K. Srinivasan, Single Self-Assembled InAs/GaAs Quantum Dots in Photonic Nanostructures: The Role of Nanofabrication, Phys. Rev. Appl. 9, 064019 (2018).

[24] Y. Xuan, H. Lin, and P. D. Ye, Simplified surface preparation for GaAs passivation using atomic layer-deposited high- $\kappa$ dielectrics, IEEE Trans. Electron Devices 54, 1811 (2007).

[25] M. Rebaud, M.-C. Roure, V. Loup, P. Rodriguez, E. Martinez, and P. Besson, Chemical treatments for native oxides removal of GaAs wafers, ECS Trans. 69, 243 (2015).

[26] E. Yablonovitch, C. J. Sandroff, R. Bhat, and T. Gmitter, Nearly ideal electronic properties of sulfide coated GaAs surfaces, Appl. Phys. Lett. 51, 439 (1987).

[27] T. Ohno, Sulfur passivation of GaAs surfaces, Phys. Rev. B 44, 6306 (1991).

[28] A. V. Kuhlmann, J. Houel, A. Ludwig, L. Greuter, D. Reuter, A. D. Wieck, M. Poggio, and R. J. Warburton, Charge noise and spin noise in a semiconductor quantum device, Nat. Phys. 9, 570 (2013).

[29] J. Robertson, Y. Guo, and L. Lin, Defect state passivation at III-V oxide interfaces for complementary metal-oxide-semiconductor devices, J. Appl. Phys. 117, 112806 (2015).

[30] The measured $Q$ factors obtained with an electrically contacted passivated sample and with an electrically uncontacted passivated sample are similar. The latter are not shown in Fig. 3(b).

[31] C. K. Carniglia and D. G. Jensen, Single-layer model for surface roughness, Appl. Opt. 41, 3167 (2002).

[32] The semiconductor heterostructure without doping is not grown by the same molecular beam epitaxy system as the semiconductor heterostructure with doping.

[33] J. M. Bennett, Recent developments in surface roughness characterization, Meas. Sci. Technol. 3, 1119 (1992).

[34] J. Davies, The Physics of Low-Dimensional Semiconductors: An Introduction (Cambridge University Press, New York, 1997).

[35] The Airy function is defined as $\operatorname{Ai}(z)=1 /(2 \cdot \pi)$ $\int_{-\infty}^{\infty} e^{\left(i *\left(z * t+t^{3} / 3\right)\right)} d t$

[36] L. Zamora Peredo, L. García-González, J. Hernandez Torres, I. Cortes-Mestizo, V. Mendez-Garcia, and M. LópezLópez, Photoreflectance and Raman study of surface electric states on $\mathrm{AlGaAs} / \mathrm{GaAs}$ heterostructures, J. Spectrosc. 2016, 1 (2016).

[37] To estimate the capping fields in Ref. [36], we assume midgap pinning, dividing half the band gap $E_{g} / 2=0.71 \mathrm{eV}$ of $\mathrm{GaAs}$ at $300 \mathrm{~K}$ by the capping-layer thicknesses reported there.

[38] Reflectance spectra [Fig. 2(a)] of different samples with nominally the same dielectric coating exhibited up to 6$\mathrm{nm}$ shifts in wavelength, most probably due to thickness variations across the wafer.

[39] F. Beauville, The VIRGO oration, The VIRGO large mirrors: A challenge for low loss coatings, Class. Quantum Gravity 21, S935 (2004). 